\title{
Exact dynamical behavior for a dual Kaup-Boussinesq system by symmetry reduction and coupled trial equations method
}

Wen-He Li $i^{1,2}$ and Yong Wang ${ }^{1 *}$

"Correspondence:

wyhitnew@163.com

1 Department of Mathematics,

Harbin Institute of Technology,

Harbin, China

Full list of author information is

available at the end of the article

\begin{abstract}
We propose a coupled trial equation method for a coupled differential equations system. Furthermore, according to the invariant property under the translation, we give the symmetry reduction of a dual Kaup-Boussinesq system, and then we use the proposed trial equation method to construct its exact solutions which describe its dynamical behavior. In particular, we get a cosine function solution with a constant propagation velocity, which shows an important periodic behavior of the system.
\end{abstract}

Keywords: Trial equation method; Symmetry; The complete discrimination system for polynomial; Exact solution; Kaup-Boussinesq system

\section{Introduction}

The usual Kaup-Boussinesq system which reads

$$
\left\{\begin{array}{l}
u_{t}=u_{x x x}+2(u v)_{x}, \\
v_{t}=u_{x}+v v_{x}
\end{array}\right.
$$

describes the motion of water wave, where $u(x, t)$ is the height of the water surface above a horizontal bottom and $v(x, t)$ is the horizontal velocity. The solutions of the nonlinear system have been studied from several aspects, and some interesting phenomena have been discovered [1-6]. For example, Smirnov obtained its real finite-gap regular solution [1], Borisov et al. studied its proliferation scheme [2], Kamchatnov et al. constructed the asymptotic soliton train solutions [3], and so on.

In general, it is difficult to study the exact dynamical behavior for nonlinear evolution problems. Therefore, some powerful methods, such as Ma and Lee's transformed rational function method [7], Liu's canonical-like transformation method [8] and trial equation method [9-14], Ma and Zhu's multiple exp-function method [15], and other direct expansion methods [16], and so forth, have been proposed to solve such problems. On the other hand, we can use the complete discrimination system for polynomial method to classify exact solutions for some nonlinear differential equations [17-25]. These methods have been extensively developed and applied to a lot of nonlinear problems [26-37]. Liu's

(c) The Author(s) 2019. This article is distributed under the terms of the Creative Commons Attribution 4.0 International License (http://creativecommons.org/licenses/by/4.0/), which permits unrestricted use, distribution, and reproduction in any medium, provided you give appropriate credit to the original author(s) and the source, provide a link to the Creative Commons license, and indicate if changes were made. 
renormalization method and its applications can be found in [38-45]. Some new methods and results on fractional differential equations can be seen in [46-57] and the references therein.

In the paper, we will study a dual Kaup-Boussinesq system. By proposing a coupled trial equation method and using symmetry reduction and a complete discrimination system for polynomial, we obtain its exact solutions which describe the dynamical behavior of the system. In particular, we find a cosine function solution which shows an important periodic motion.

This paper is organized as follows. In Sect. 2, we propose a coupled trial equation method. In Sect. 3, we give the reduction of the dual Kaup-Boussinesq system according to the symmetry property and the proposed trial equation method. In Sect. 4, we give the exact solutions by using the complete discrimination system for polynomial. In particular, we get an interesting periodic cosine solution. The last section is a short conclusion.

\section{Trial equation method for a coupled system}

We propose a generalization of Liu's trial equation method [9-14] to coupled differential equations systems as follows. Consider the coupled system:

$$
\begin{aligned}
& N_{1}\left(u, v, u_{t}, v_{t}, u_{x}, v_{x}, \ldots\right)=0, \\
& N_{2}\left(u, v, u_{t}, v_{t}, u_{x}, v_{x}, \ldots\right)=0 .
\end{aligned}
$$

Under the traveling wave transformation $u(x, t)=u(\xi), v(x, t)=v(\xi)$, where $\xi=x-c t$, the above system of equations becomes a coupled ordinary differential equations system

$$
\begin{aligned}
& M_{1}\left(u, v, u^{\prime}, v^{\prime}, \ldots\right)=0, \\
& M_{2}\left(u, v, u^{\prime}, v^{\prime}, \ldots\right)=0 .
\end{aligned}
$$

We take trial equations as follows:

$$
\begin{aligned}
& u^{\prime}=H(u), \\
& v=G(u),
\end{aligned}
$$

or

$$
\begin{aligned}
& v^{\prime}=H(v), \\
& u=G(v),
\end{aligned}
$$

where $H$ and $G$ are two unknown functions which need to be determined. Substituting these trial equations into the coupled system, we solve $H$ and $G$, and then integrate the trial equation (5) or (7) to give the corresponding exact solutions such as

$$
\xi-\xi_{0}=\int \frac{\mathrm{d} u}{H(u)}, \quad v=G(u) .
$$

If $H$ is a polynomial, we will use the complete discrimination system for polynomial to classify the exact solutions. In the next section, we give the application of the proposed trial equation method to a dual Kaup-Boussinesq system. 


\section{Symmetry and reduction}

The considered new dual Kaup-Boussinesq (for simplicity, KB) system is taken as follows:

$$
\left\{\begin{array}{l}
u_{x x t}+\left(u u_{x x}+u_{x}^{2}+u^{2}+2 u v\right)_{x}=0 \\
v_{t}+(u v)_{x}=0
\end{array}\right.
$$

By taking the traveling wave transformation $u=u(\xi), v=v(\xi), \xi=x-c t$, we have

$$
\left\{\begin{array}{l}
-c u^{\prime \prime \prime}+\left(u u^{\prime \prime}+\left(u^{\prime}\right)^{2}+u^{2}+2 u v\right)^{\prime}=0 \\
-c v^{\prime}+(u v)^{\prime}=0
\end{array}\right.
$$

It is easy to see that the above equations are invariant under the translational transformation of $\xi$, so we can reduce the system. Substituting trial equations (5) and (6) into the above system and integrating them yield

$$
\left\{\begin{array}{l}
(u-c) H^{\prime} H+H^{2}+u^{2}+2 u v=c_{1}, \\
v=G(u)=\frac{c_{2}}{u-c},
\end{array}\right.
$$

where $c_{1}$ and $c_{2}$ are two arbitrary constants. And then the above system becomes

$$
(u-c) H^{\prime} H+H^{2}+u^{2}+\frac{2 u c_{2}}{u-c}=c_{1} .
$$

Furthermore, we take the following transformation:

$$
H^{2}=W,
$$

and get

$$
W^{\prime}(u)+\frac{2}{u-c} W(u)=\frac{2\left(c_{1}-u^{2}-\frac{2 u c_{2}}{u-c}\right)}{u-c} .
$$

This is a first order linear non-homogeneous differential equation whose general solution is given by

$$
\begin{aligned}
W(u) & =e^{-\int \frac{2}{u-c} \mathrm{~d} u}\left\{\int 2 \frac{c_{1}-u^{2}-2 u \frac{c_{2}}{u-c}}{u-c} e^{\int \frac{2}{u-c} \mathrm{~d} u} \mathrm{~d} u+c_{3}\right\} \\
& =\frac{-\frac{1}{2} u^{4}+\frac{2}{3} c u^{3}+\left(2 c_{1}-2 c_{2}\right) u^{2}-2 c c_{1} u+c_{3}}{(u-c)^{2}},
\end{aligned}
$$

that is,

$$
\left(u^{\prime}\right)^{2}=H^{2}(u)=\frac{-\frac{1}{2} u^{4}+\frac{2}{3} c u^{3}+\left(2 c_{1}-2 c_{2}\right) u^{2}-2 c c_{1} u+c_{3}}{(u-c)^{2}},
$$

which is just our needed result. Rewrite it as the form of elementary integrals

$$
\pm \sqrt{\frac{1}{2}}\left(\xi-\xi_{0}\right)=\int \frac{(u-c) \mathrm{d} u}{\sqrt{-\left(u^{4}-\frac{4}{3} c u^{3}-\left(4 c_{1}-4 c_{2}\right) u^{2}-4 c c_{1} u+2 c_{3}\right)}},
$$


from which we can give exact solutions. In fact, we can further simplify it by taking

$$
\omega=u-\frac{1}{3} c
$$

and hence

$$
\pm \sqrt{\frac{1}{2}}\left(\xi-\xi_{0}\right)=\int \frac{\left(\omega-\frac{2}{3} c\right) \mathrm{d} \omega}{\sqrt{-\left(\omega^{4}+p \omega^{2}+q \omega+r\right)}}
$$

where

$$
\begin{aligned}
& p=-\frac{7}{9} c^{2}-4 c_{1}+4 c_{2}, \quad q=\frac{1}{9} c^{2}-\frac{8}{27} c^{3}-\frac{20}{3} c_{1} c+\frac{8}{3} c_{2} c, \\
& r=\frac{20}{9} c_{1} c+\frac{4}{9} c_{2} c^{2}+2 c_{3} .
\end{aligned}
$$

In the next section, we give exact solutions for the KB coupled system (10) according to the above integral (20).

Remark 1 If we consider $\left(u^{\prime}\right)^{2}$ as kinetic energy and the right-hand side of (17) as negative potential energy, then (17) gives a first integral, that is, conservation of energy.

\section{Exact solutions}

Denote $F(\omega)=\omega^{4}+p \omega^{2}+q \omega+r$. Then its complete discrimination system is given by [25]

$$
\left\{\begin{array}{l}
D_{1}=4 \\
D_{2}=-p \\
D_{3}=-2 p^{3}+8 p r-9 q^{2} \\
D_{4}=-p^{3} q^{2}+4 p^{4} r+36 p q^{2} r-32 p^{2} r^{2}-\frac{27}{4} q^{4}+64 r^{3} \\
E_{2}=9 q^{2}-32 p r
\end{array}\right.
$$

According to the discrimination system [25], and considering the special form of integrand in (20), we have the following four families of solutions.

Family $1 D_{4}=0, D_{3}>0, D_{2}>0$. Then we have

$$
F(\omega)=(\omega-\alpha)^{2}(\omega-\beta)(\omega-\gamma),
$$

where $\alpha, \beta, \gamma$ are real numbers, and $\beta>\gamma$. When $\gamma<\alpha<\beta$, we have

$$
\pm \sqrt{\frac{1}{2}}\left(\xi-\xi_{0}\right)=\frac{2 \alpha-c}{\sqrt{(\beta-\alpha)(\gamma-\alpha)}} \arctan \sqrt{\frac{\alpha-\beta}{\gamma-\alpha} \frac{u+\frac{c}{3}-\gamma}{\beta-u-\frac{c}{3}}}+2 \arctan \sqrt{\frac{u+\frac{c}{3}-\gamma}{\beta-u-\frac{c}{3}}}
$$

and when $\alpha>\beta$ and $w>\beta$, or when $\alpha<\gamma$ and $w<\gamma$, we have

$$
\pm \sqrt{\frac{1}{2}}\left(\xi-\xi_{0}\right)=\frac{2 \alpha-c}{\sqrt{(\alpha-\beta)(\gamma-\alpha)}} \ln \frac{\sqrt{\frac{\alpha-\beta}{\gamma-\alpha} \frac{u+\frac{c}{3}-\gamma}{\beta-u-\frac{c}{3}}}+1}{\sqrt{\frac{\alpha-\beta}{\gamma-\alpha} \frac{u+\frac{c}{3}-\gamma}{\beta-u-\frac{c}{3}}}-1}+2 \arctan \sqrt{\frac{u+\frac{c}{3}-\gamma}{\beta-u-\frac{c}{3}}}
$$


Family $2 D_{4}=0, D_{3}=0, D_{2}>0, E_{2}=0$. Then we have

$$
F(\omega)=(\omega-\alpha)^{3}(\omega-\beta),
$$

where $\alpha, \beta$ are real numbers. The solution is given by

$$
\pm \sqrt{\frac{1}{2}}\left(\xi-\xi_{0}\right)=\frac{2 \alpha-c}{\alpha-\beta} \sqrt{\frac{u+\frac{c}{3}-\gamma}{\beta-u-\frac{c}{3}}}+2 \arctan \sqrt{\frac{u+\frac{c}{3}-\gamma}{\beta-u-\frac{c}{3}}}
$$

Family $3 D_{4}>0, D_{3}>0, D_{1}>0$. Then we have

$$
F(\omega)=\left(\omega-\alpha_{1}\right)\left(\omega-\alpha_{2}\right)\left(\omega-\alpha_{3}\right)\left(\omega-\alpha_{4}\right),
$$

where $\alpha_{1}, \alpha_{2}, \alpha_{3}, \alpha_{4}$ are real numbers, and $\alpha_{1}>\alpha_{2}>\alpha_{3}>\alpha_{4}$. The solutions can be represented in terms of the first and second kinds of elliptic integrals. We can also give more simple forms by taking the transformation

$$
\omega_{1}=\frac{A^{\frac{1}{3}}}{\omega-\alpha_{1}}
$$

where $A=\left(\alpha_{1}-\alpha_{2}\right)\left(\alpha_{1}-\alpha_{3}\right)\left(\alpha_{1}-\alpha_{4}\right)$. The corresponding integral becomes

$$
\begin{aligned}
\pm \sqrt{\frac{1}{2}}\left(\xi-\xi_{0}\right)= & \int \frac{\mathrm{d} \omega_{1}}{\omega_{1} \sqrt{-\left(\omega_{1}-\beta_{1}\right)\left(\omega_{1}-\beta_{2}\right)\left(\omega_{1}-\beta_{3}\right)}} \\
& +\int \frac{\left(\alpha_{1}-\frac{2 c}{3}\right) A^{-\frac{1}{3}} \mathrm{~d} \omega_{1}}{\omega_{1} \sqrt{-\left(\omega_{1}-\beta_{1}\right)\left(\omega_{1}-\beta_{2}\right)\left(\omega_{1}-\beta_{3}\right)}}
\end{aligned}
$$

where $\beta_{1}=\frac{A^{\frac{1}{3}}}{\alpha_{4}-\alpha_{1}}, \beta_{2}=\frac{A^{\frac{1}{3}}}{\alpha_{3}-\alpha_{1}}, \beta_{3}=\frac{A^{\frac{1}{3}}}{\alpha_{2}-\alpha_{1}}$.

Family $4 D_{4}<0, D_{2} D_{3} \geq 0$. Then we have

$$
F(\omega)=(\omega-\alpha)(\omega-\beta)\left(\left(\omega-l_{1}\right)^{2}+s_{1}^{2}\right),
$$

where $\alpha, \beta, l_{1}$, and $s_{1}$ are real numbers, and $\alpha>\beta>, s_{1}>0$. The solutions can be represented in terms of the first and second kinds of elliptic integrals.

From Remark 1, we know that the above result gives the classification of all solutions of integral (20).

Remark 2 Here we only write the expressions of $u$, by which $v$ can be given from (4). For simplicity, we omit $v$.

In particular, we find that there is an interesting periodic cosine function solution although all the above solutions are implicit forms. Indeed, if we take the wave velocity to 
be a constant $c=2 \alpha$, we get the periodic solution

$$
u=\beta-\frac{2 \alpha}{3}+\left(\frac{\alpha}{3}-\beta-\gamma\right) \cos ^{2}\left(\frac{\xi-\xi_{0}}{2 \sqrt{2}}\right)
$$

where $\xi=x-\frac{2 \alpha}{3} t$. This is a periodic traveling wave with constant velocity.

In fact, we only need to take the first term to be zero in solution (24) or (25) or (27), and then we have

$$
\pm \sqrt{\frac{1}{2}}\left(\xi-\xi_{0}\right)=2 \arctan \sqrt{\frac{u+\frac{c}{3}-\gamma}{\beta-u-\frac{c}{3}}},
$$

by which we get the above periodic solution.

From solution (32), we know that the dual KB system shows an important periodic dynamical behavior.

\section{Conclusion}

A dual Kaup-Boussinesq system is solved by symmetry reduction and a coupled trial equation method. The result includes four families of exact single traveling wave solutions for this system. Among those, if we consider $\xi$ as the functions of $u$ or $v$ respectively, the solutions are given by the explicit functions, and reversely, the solutions are represented by implicit functions. In particular, when the wave propagation velocity is taken as a special constant, the KB system has a periodic cosine function solution. This solution shows an important periodic dynamical behavior. In summary, according to these exact solutions, a variety of evolution patterns for the coupled KB system are obtained.

\section{Acknowledgements}

The authors thank the reviewers for their helpful suggestions.

\section{Funding}

The first author is supported by the project of Youth Fund of Northeast Petroleum University (No. 2019QNL-38).

Availability of data and materials

Not applicable.

\section{Competing interests}

The authors declare that they have no competing interests.

\section{Authors' contributions}

WL completed the whole computation and wrote the draft, YW proposed the topic. Both authors read and approved the final manuscript.

\section{Author details}

${ }^{1}$ Department of Mathematics, Harbin Institute of Technology, Harbin, China. ${ }^{2}$ Department of Mathematics, Northeast Petroleum University, Daqing, China.

\section{Publisher's Note}

Springer Nature remains neutral with regard to jurisdictional claims in published maps and institutional affiliations.

Received: 12 July 2019 Accepted: 27 September 2019 Published online: 26 October 2019

\section{References}

1. Smirnov, A.O.: Real finite-gap regular solutions of the Kaup-Boussinesq equation. Theor. Math. Phys. 66(1), 19-31 (1986)

2. Borisov, A.B., Pavlov, M.P., Zykov, S.A.: Proliferation scheme for Kaup-Boussinesq system. Physica D 152, 104-109 (2001) 
3. Kamchatnov, A.M., Kraenkel, R.A., Umarov, B.A.: Asymptotic soliton train solutions of Kaup-Boussinesq equations. Wave Motion 38(4), 355-365 (2003)

4. Bhrawy, A.H., Tharwat, M.M., Abdelkawy, M.A.: Integrable system modelling shallow water waves: Kaup-Boussinesq shallow water system. Indian J. Phys. 87(7), 665-671 (2013)

5. Hosseini, K., Ansari, R., Gholamin, P.: Exact solutions of some nonlinear systems of partial differential equations by using the first integral method. J. Math. Anal. Appl. 387(2), 807-814 (2012)

6. Zhou, J., Tian, L., Fan, X.: Solitary-wave solutions to a dual equation of the Kaup-Boussinesq system. Nonlinear Anal., Real World Appl. 11(4), 3229-3235 (2010)

7. Ma, W.X., Lee, J.H.: A transformed rational function method and exact solutions to the $3+1$ dimensional Jimbo-Miwa equation. Chaos Solitons Fractals 42(3), 1356-1363 (2009)

8. Liu, C.S.: Canonical-like transformation method and exact solutions to a class of diffusion equations. Chaos Solitons Fractals 42(1), 441-446 (2009)

9. Liu, C.S.: Trial equation method and its applications to nonlinear evolution equations. Acta Phys. Sin. 54(6), 2505-2509 (2005)

10. Liu, C.S.: Using trial equation method to solve the exact solutions for two kinds of KdV equations with variable coefficients. Acta Phys. Sin. 54(10), 4506-4510 (2005)

11. Liu, C.S.: Trial equation method for nonlinear evolution equations with rank inhomogeneous: mathematica discussions and applications. Commun. Theor. Phys. 45(2), 219-223 (2006)

12. Liu, C.S.: A new trial equation method and its applications. Commun. Theor. Phys. 45(3), 395-397 (2006)

13. Liu, C.S.: Trial equation method based on symmetry and applications to nonlinear equations arising in mathematical physics. Found. Phys. 41(5), 793-804 (2011)

14. Liu, C.S.: Exponential function rational expansion method for nonlinear differential-difference equations. Chaos Solitons Fractals 40, 708-716 (2009)

15. Ma, W.X., Zhu, Z:: Solving the (3+1)-dimensional generalized KP and BKP equations by the multiple exp-function algorithm. Appl. Math. Comput. 218(24), 11871-11879(2012)

16. Wazwaz, A.M.: Partial Differential Equations and Solitary Waves Theory. Springer, Berlin (2010)

17. Liu, C.S.: New exact envelope traveling wave solutions to higher-order dispersive cubic-qintic nonlinear Schrödinger equation. Commun. Theor. Phys. 44(5), 799-801 (2015)

18. Liu, C.S.: Travelling wave solutions of triple sine-Gordon equation. Chin. Phys. Lett. 21(12), 2369-2371 (2004)

19. Liu, C.S.: Solution of ODE $u^{\prime \prime}+p(u)\left(u^{\prime}\right)^{2}+q(u)=0$ and applications to classifications of all single travelling wave solutions to some nonlinear mathematical physics equations. Commun. Theor. Phys. 49(2), 291-296 (2008)

20. Liu, C.S.: New exact envelope traveling wave solutions to higher-order dispersive cubic-qintic nonlinear Schrödinger equation. Commun. Theor. Phys. 44(5), 799-801 (2005)

21. Liu, C.S.: All single traveling wave solutions to $(3+1)$-dimensional Nizhnok-Novikov-Veselov equation. Commun. Theor. Phys. 45(6), 991-992 (2006)

22. Liu, C.S.: Travelling wave solutions of a kind of generalized Ginzburg-Landau equation. Commun. Theor. Phys. 43(4), 787-790 (2005)

23. Liu, C.S.: Classification of all single travelling wave solutions to Calogero-Degasperis-Focas equation. Commun. Theor. Phys. 48(4), 601-604 (2007)

24. Liu, C.S.: Travelling wave solutions to $1+1$ dimensional dispersive long wave equation. Chin. Phys. 14(9), 1710-1715 (2015)

25. Liu, C.S.: Applications of complete discrimination system for polynomial for classifications of traveling wave solutions to nonlinear differential equations. Comput. Phys. Commun. 181(2), 317-324 (2010)

26. Yang, S.: The envelope travelling wave solutions to the Gerdjikov-Ivanov model. Pramana 91(3), 36 (2018)

27. Yang, S.: Exact solutions to Zakharov-Kuznetsov equation with variable coefficients by trial equation method. Z. Naturforsch. A 73(1), 1-4 (2017)

28. Mirzazadeh, M.: Soliton solutions of Davey-Stewartson equation by trial equation method and ansatz approach. Nonlinear Dyn. 82(4), 1775-1780 (2016)

29. Liu, Y.: Exact solutions to nonlinear Schrödinger equation with variable coefficients. Appl. Math. Comput. 217(12), $5866-5869$ (2011)

30. Wang, X., Liu, Y.: All envelop traveling wave patterns to nonlinear Schrödinger equation in parabolic law medium. Mod. Phys. Lett. B 33(1), 1850428 (2019)

31. Wang, X., Liu, Y.: All single travelling wave patterns to fractional Jimbo-Miwa equation and Zakharov-Kuznetsov equation. Pramana 92(3), 31 (2019)

32. Liu, Y., Wang, X.: The construction of solutions to Zakharov-Kuznetsov equation with fractional power nonlinear terms. Adv. Differ. Equ. 2019(1), 134 (2019)

33. Zheng, B., Kai, Y., Xu, W., Yang, N., Zhang, K., Thibado, P.M.: Exact traveling and non-traveling wave solutions of the time fractional reaction-diffusion equation. Phys. A, Stat. Mech. Appl. 532(15), 121780 (2019)

34. Mao, Y.: Exact solutions to $2+1$-dimensional Chaffee-Infante equation. Pramana 91(1), 9 (2018)

35. Triki, H., Wazwaz, A.M.: Trial equation method for solving the generalized Fisher equation with variable coefficients. Phys. Lett. A 380(13), 1260-1262 (2016)

36. Chen, S., Liu, Y., Wei, L., Guan, B.: Exact solutions to fractional Drinfeld-Sokolov-Wilson equations. Chin. J. Phys. 56(2), 708-720 (2018)

37. Ozyapici, A., Bilgehan, B.: Generalized system of trial equation methods and their applications to biological systems. Appl. Math. Comput. 338, 722-732 (2018)

38. Liu, C.: The essence of the generalized Newton binomial theorem. Commun. Nonlinear Sci. Numer. Simul. 15(10), $2766-2768(2010)$

39. Wang, C.Y.: The analytic solutions of Schrodinger equation with cubic-quintic nonlinearities. Results Phys. 10 , 150-154 (2018)

40. Liu, C.: The renormalization method based on the Taylor expansion and applications for asymptotic analysis. Nonlinear Dyn. 88(2), 1099-1124 (2017)

41. Liu, C.: The renormalization method from continuous to discrete dynamical systems: asymptotic solutions, reductions and invariant manifolds. Nonlinear Dyn. 94(2), 873-888 (2018) 
42. Kai, Y., Zheng, B., Zhang, K., Xu, W., Yang, N.: Exact and asymptotic solutions to magnetohydrodynamic flow over a nonlinear stretching sheet with a power-law velocity by the homotopy renormalization method. Phys. Fluids 31(6), $063606(2019)$

43. Wang, C.Y: Asymptotic analysis to Von Karman swirling-flow problem. Mod. Phys. Lett. B 33(25), 1950298 (2019)

44. Kai, Y., Zheng, B.: Asymptotic analysis to free-convective boundary-layer problem by homotopy renormalization method. Mod. Phys. Lett. B 33(7), 1950083 (2019)

45. Kai, Y.: Exact solutions and asymptotic solutions of one-dimensional domain walls in nonlinearly coupled system. Nonlinear Dyn. 92(4), 1665-1677 (2018)

46. Gao, F., Yang, X.J., Srivastava, H.M.: Exact traveling-wave solutions for linear and non-linear heat transfer equations. Therm. Sci. 21(6), 2307-2311 (2017)

47. Yang, X.J., Tenreiro Machado, J.A., Baleanu, D., Cattani, C.: On exact traveling-wave solutions for local fractional Korteweg-de Vries equation. Chaos 26(8), 084312 (2016)

48. Yang, X.J., Gao, F., Srivastava, H.M.: Exact travelling wave solutions for the local fractional two-dimensiona Burgers-type equations. Comput. Math. Appl. 73(2), 203-210 (2017)

49. Yang, X.J: A new integral transform operator for solving the heat-diffusion problem. Appl. Math. Lett. 64, 193-197 (2017)

50. Yang, X.J.: New integral transforms for solving a steady heat transfer problem. Therm. Sci. 21(1), S79-S87 (2017)

51. Yang, X.J., Gao, F.: A new technology for solving diffusion and heat equations. Therm. Sci. 21(1A), 133-140 (2017)

52. Zhang, X., Agarwal, P., Liu, Z., Peng, H.: The general solution for impulsive differential equations with Riemann-Liouville fractional-order $q \in(1,2)$. Open Math. 13(1), 908-930 (2015)

53. Agarwal, P., Dragomir, S.S., Jeli, M., Samet, B.: Advances in Mathematical Inequalities and Applications. Birkhäuser, Basel (2019)

54. Rekhviashvili, S., Pskhu, A., Agarwal, P., Jain, S.: Application of the fractional oscillator model to describe damped vibrations. Turk. J. Phys. 43(3), 236-242 (2019)

55. Sitho, S., Ntouyas, S.K., Agarwal, P., Tariboon, J.: Noninstantaneous impulsive inequalities via conformable fractional calculus. J. Inequal. Appl. 2018(1), 261 (2018)

56. Saoudi, K., Agarwal, P., Kumam, P., Ghanmi, A., Thounthong, P.: The Nehari manifold for a boundary value problem involving Riemann-Liouville fractional derivative. Adv. Differ. Equ. 2018(1), 263 (2018)

57. Hammouch, Z., Mekkaoui, T., Agarwal, P.: Optical solitons for the Calogero-Bogoyavlenskii-Schiff equation in $(2+1)$ dimensions with time-fractional conformable derivative. Eur. Phys. J. Plus 133(7), 248 (2018)

\section{Submit your manuscript to a SpringerOpen ${ }^{\circ}$ journal and benefit from:}

- Convenient online submission

- Rigorous peer review

- Open access: articles freely available online

- High visibility within the field

- Retaining the copyright to your article

Submit your next manuscript at $\boldsymbol{~ s p r i n g e r o p e n . c o m ~}$ 